\title{
COMPUTER SIMULATIONS FOR THE PREDICTION OF MICROSTRUCTURE/PROPERTY VARIATION IN AEROTURBINE DISKS
}

\author{
Herng-Jeng Jou ${ }^{1}$, Peter Voorhees ${ }^{2}$, Gregory B. Olson ${ }^{2}$ \\ ${ }^{1}$ QuesTek Innovations LLC, 1820 Ridge Avenue, Evanston, IL 60201, USA, email: hjjou@questek.com \\ ${ }^{2}$ Materials Science and Engineering, Northwestern University, 2220 Campus Drive, Evanston, IL 60208, USA
}

Keywords: Precipitation, Nucleation, Growth, Coarsening, Multicomponent, CALPHAD, IN100, Superalloys, AIM

\begin{abstract}
In support of a new methodology of accelerated development and qualification of new materials, the PrecipiCalc ${ }^{\mathrm{TM}}$ software has been developed as an efficient, high-fidelity 3D multiphase precipitation simulator grounded in computational thermodynamics and multicomponent diffusional nucleation and growth models incorporating interfacial dissipations. With extensive validation under a major federally sponsored program, the software has been successfully applied to the accurate and rapid simulation of multiscale (from $\mathrm{nm}$ to $\mu \mathrm{m}$ ), multimodal $\gamma^{\prime}$ microstructure development in IN100 Ni-based aeroturbine disk alloys under complex commercial heattreatment processing. Integration of PrecipiCalc with FEM heat transfer simulation and a strength model has demonstrated accurate prediction of property variation for accelerated process optimization and probabilistic modeling of design minimum properties.
\end{abstract}

\section{Introduction}

The recently completed 3-year DARPA-AIM program on Accelerated Insertion of Materials has successfully demonstrated a new methodology of integrated computational simulation and focused testing, to rapidly optimize materials processing at the component level and efficiently predict design minimum properties through mechanisticallybased probabilistic methods. As a central tool of this methodology, the PrecipiCalc software was developed under the AIM initiative to provide rapid high-fidelity simulation of microstructural evolution during thermal processing. To support efficient property prediction, the code is structured to predict the microstructural parameters necessary for state-of-the-art structure/property relations, incorporating only the physics necessary for required accuracy.

Under the AIM initiative, a high degree of simulation fidelity was achieved through intensive iterative interaction of theory and experiment through microstructural characterization of Ni-based IN100 aeroturbine disks over a wide range of scales. Various experimental techniques were used to characterize the IN100 samples taken from the aeroturbine disks after commercial heat treatment, including TEM for microstructural morphology of precipitations and EDS/3DAP for compositions of both precipitates and matrix. Major microstructural constituents in IN100 are $\gamma$ (FCC) matrix and $\gamma^{\prime}$ precipitates, as well as small amounts of carbide and boride phases. For this study, we focused on a 3-step heat treatment cycle for the IN100 aeroturbine disk, and the cycle includes: (1) $1143^{\circ} \mathrm{C}$ solution (subsolvus) treatment, followed by a transfer and oil quench; (2) $982^{\circ} \mathrm{C}$ heat treatment followed by fan cool; and (3) $732^{\circ} \mathrm{F}$ heat treatment followed by fan cool.

The 3-step heat treatment cycle produces a final microstructure with three distributions of $\gamma^{\prime}$ precipitates at distinct sizes, named primary (large), secondary (intermediate) and tertiary (small), with a total fraction of about $60 \%$. Figure 1 provides two example micrographs of IN100 $\gamma^{\prime}$ microstructure. Primary $\gamma^{\prime}$ particles are formed in the powder extrusion and forging process, and are coarsened at subsolvus solution temperature, providing grain pinning to matrix grain structure. The secondary (tertiary) $\gamma^{\prime}$ develops from the quench after the first (second) treatment temperature and coarsens in the second (third) heat treatment step. Fine $\gamma^{\prime}$ particles have a much higher number density than the coarse ones. As shown in Figure 1, each primary (and secondary) particle is surrounded by many secondary (tertiary) particles.

This article discusses the effort under the AIM program to mechanistically capture the $\gamma^{\prime}$ precipitation microstructure in the IN100 disk alloy. We will cover the PrecipiCalc multicomponent precipitation models, software implementation techniques, and the applications to the IN100 alloys.

\section{Multicomponent Precipitation Models}

Precipitation is one of the most important types of phase transformation processes in material systems. It changes material properties in many ways and is often critical to the material performance. The ability to control the microstructure of precipitates provides a powerful tool in material development. Due to its importance, precipita- 

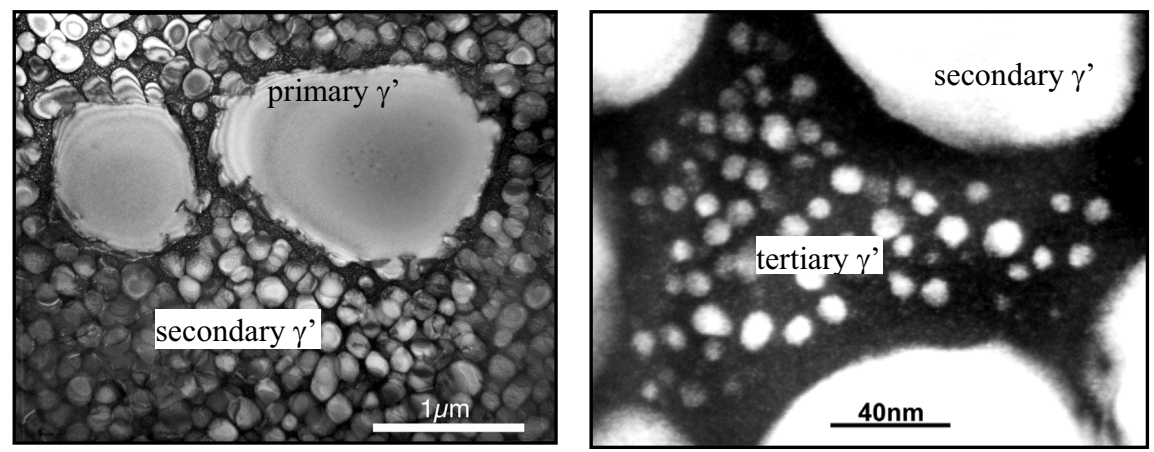

Figure 1: Experimental micrographs of IN100 at two resolutions clearly show three distinct size distributions of $\gamma^{\prime}$ (courtesy Gautam Ghosh, Northwestern University).

tion modeling has been an active research area in materials science. Precipitation generally includes three physical mechanisms: nucleation, growth and coarsening. Each of them has received extensive research in the past. In the continuum modeling arena, different approaches to the precipitation model have been proposed, involving different levels of approximation and computational complexity. From the Avrami-type single state variable (usually volume fraction) models to complex phase-field models, the computational expense scales exponentially with the details captured by the models.

PrecipiCalc precipitation models are based on the foundation of the double state variable approach, following the framework of Langer-Schwartz (LS) [1], which provided an integrated approach of nucleation, growth and coarsening, and Kampmann-Wagner (KW) [2], which provided a numerical solution to LS model. The two state variables are particle size and number density, and together they define the particle size distribution (PSD). With the PSD, higher level quantities such as volume fractions, mean sizes and interparticle distances can be calculated. Within this framework, the key models are nucleation (source of new particles), growth (particle size changes) under mean field, continuity (time evolution of PSD), and mass balance. Coarsening comes out naturally within this framework. Due to the mechanistic integration of physical mechanisms in LS framework, the transitions between mechanisms are captured without ad hoc treatments. For example, high supersaturation precipitation in an alloy system can show only nucleation and coarsening, without an intermediate growth stage, which has been captured by the LS model.

The adoption of numerical LS-KW framework in modeling precipitation has been successfully applied to many alloy systems $[5,6,7,8]$. However, those work have only provided the models for binary systems, and the applications to multicomponent systems require the reduction to pseudo-binary systems. The true multicomponent treat- ment within the LS-KW framework is limited. The work of Popov [9, 10] dealt with the multicomponent growth and evolution of PSD, without nucleation. In the coarsening region, several multicomponent models [12, 13, 14, 15] have been proposed. The complete model and implementation of integrated nucleation, growth and coarsening for multicomponent systems are not available. Furthermore, many of the mentioned literature work focused on isothermal conditions. In complex commercial heat treatment of alloys, it is important to model precipitation under nonisothermal conditions.

In the following subsections, detailed PrecipiCalc multicomponent precipitation models are presented.

\section{Continuity Equation}

The particle size distribution, $f(R, t)$ is defined such that $f(R, t) d R$ is the number of particles per unit volume of size $R$ to $R+d R$. Thus, the number of particles per unit volume in the sample, $N_{v}$, is

$$
N_{v}=\int_{0}^{\infty} f(R, t) d R
$$

In our case, the volume fraction of particles, $\phi$, is

$$
\phi=\int_{0}^{\infty} \frac{4 \pi}{3} f(R, t) R^{3} d R
$$

The particle size distribution evolves in time according to:

$$
\frac{\partial f}{\partial t}+\frac{\partial(f d R / d t)}{\partial R}=I(R, t)
$$

where $I(R, t)$ is the nucleation rate.

\section{Growth Model}

The growth rate is determined by the diffusion field surrounding the particle within the approximation of local equilibrium at the particle-matrix interface. We employ 
the quasistationary approximation and assume that the diffusion coefficient is independent of composition. Unlike binary alloys, the compositions at the interface are not fixed by the phase diagram, but are functions of the diffusion coefficients and supersaturation. We determine these compositions self consistently within the small supersaturation approximation. We also allow for capillarity, interfacial kinetics, and non-zero volume fraction correction. The growth rate of a particle of size $R$ is given by,

$$
\begin{aligned}
\frac{d R}{d t}= & \left(1+R \sqrt{4 \pi N_{v} \bar{R}}\right) \\
& \frac{\overline{\Delta C_{i} G_{i j}^{\alpha} \Delta C_{j}^{\infty}+\bar{C}_{\delta}^{\beta}\left(\bar{\mu}_{\delta}^{\alpha}-\bar{\mu}_{\delta}^{\beta}\right)-2 V_{m}^{\beta} \sigma / R}}{R \overline{\Delta C}_{i} G_{i j}^{\alpha} D_{j k}^{-1} \mathcal{B}_{k}+1 / M}(4)
\end{aligned}
$$

where the sum over repeated Latin indices from $2 \ldots N$ and repeated Greek indices from $1 \ldots N$ is assumed, $\overline{\Delta C}_{i}=\bar{C}_{i}^{\beta}-\bar{C}_{i}^{\alpha}, \bar{C}$ is the mole fraction of the denoted phase about which the free energy function is expanded, $G_{i j}=\partial^{2} G / \partial C_{i} \partial C_{j}$ evaluated at $\bar{C}_{i}, G$ is the molar Gibbs free energy of the noted phase, $\Delta C_{j}^{\infty}=C_{j}^{\infty}-\bar{C}_{j}^{\alpha}, C_{j}^{\infty}$ is the composition of component $j$ at an infinite distance from the spherical particle, $\bar{\mu}_{i}$ is the chemical potential of component $i$ evaluated at $\bar{C}_{i} i>1, V_{m}^{\beta}$ is the molar volume of the particle phase $\beta, \sigma(R)$ is the size dependent interfacial energy, $D_{j k}$ is the diffusion tensor, $R_{g}$ is the gas constant, $\bar{R}$ is the mean size, $M$ is the interfacial mobility,

$$
\begin{gathered}
\mathcal{B}_{k}=L_{k}+\overline{\Delta C}_{k}+\left(\delta_{k j}-A_{k j}\right) A_{j l}^{-1} \Delta C_{l}^{\infty}- \\
\frac{y\left(\delta_{k j}-A_{k j}\right) A_{j h}^{-1} D_{h l}^{-1} \overline{\Delta C}_{l}}{\left(\overline{\Delta C}_{j} G_{j i}^{\alpha} D_{i k}^{-1} \overline{\Delta C}_{k}\right)} \\
L_{k}=G_{j k}^{\beta-1}\left(\tilde{\mu}_{j}^{\alpha}-\tilde{\mu}_{j}^{\beta}\right)-2 G_{j k}^{\beta-1} \overline{\Delta V}_{j} \sigma / R \\
A_{k j}=G_{k l}^{\alpha-1} G_{l j}^{\beta} \\
y=\overline{\Delta C}_{i} G_{i j}^{\alpha} \Delta C_{j}^{\infty}+\bar{C}_{\delta}^{\beta}\left(\bar{\mu}_{\delta}^{\alpha}-\bar{\mu}_{\delta}^{\beta}\right)-2 V_{m}^{\beta} \sigma / R
\end{gathered}
$$

$\tilde{\mu}_{i}=\bar{\mu}_{i}-\bar{\mu}_{1}, i=2 \ldots N$, and $\overline{\Delta V}_{i}=\bar{V}_{i}-\bar{V}_{1}, i=2 \ldots N$.

Interfacial kinetics is included by assuming the interfacial velocity is proportional to the interfacial mobility $M$ and total chemical driving force on the interface. $M$ is expressed as $M_{0} \exp \left(-Q / R_{g} T\right)$ where $Q$ is the activation energy for interfacial kinetics, $R_{g}$ is the gas constant, and $M_{0}$ is the prefactor.

The composition at which the free energy is expanded is chosen such that the resulting expression for the growth rate is accurate. In a typical run this involves changing this composition, but not for every timestep. Unlike a binary, where the growth rate is a function solely of the supersaturation, in a multicomponent alloy, the growth rate depends on the supersaturation and a thermodynamic factor, $G_{, j k}$. This is because the concentrations at the interface cannot be specified by the local equilibrium conditions, but are functions of the mass balance equations at the interface.
When $y=0$ the growth rate is zero, and thus this gives an expression for the critical radius of nucleation in a multicomponent alloy,

$$
R_{c}=\frac{2 V_{m}^{\beta} \sigma}{\Delta G}
$$

where

$$
\Delta G=\overline{\Delta C}_{i} G_{i j}^{\alpha} \Delta C_{j}^{\infty}+\bar{C}_{\delta}^{\beta}\left(\bar{\mu}_{\delta}^{\alpha}-\bar{\mu}_{\delta}^{\beta}\right)
$$

the free energy change on forming $\beta$ of the composition of the critical nucleus from $\alpha$ of composition $C_{k}^{\alpha}$ where both phases are assumed incompressible at the pressure of the matrix is given by the denominator in Eq. (5). This agrees with the result obtained by the usual approach.

Homogeneous and Transient Nucleation Models

Isothermal Nucleation The nucleation rate is given by,

$$
I(R)=e^{-\tau / t} j(R)
$$

where $j(R)$ is the nucleation rate, $\tau$ is the incubation time and $t$ is time. Assume that the nucleation rate is a function of particle size,

$$
j(R)=\frac{J_{s}}{\delta \sqrt{2 \pi}} \exp \left[-\frac{\left(R-R_{c}\right)^{2}}{2 \delta^{2}}\right]
$$

where $J_{s}$ is the steady state nucleation rate, $\delta$ is the variance of the Gaussian distribution, $R_{c}$ is the critical radius of nucleation. $j(R)$ is chosen such that

$$
J_{s}=\int_{R_{c}}^{\infty} j(R) d R
$$

Thus only particles greater than the critical are considered part of the precipitate phase.

The steady state nucleation rate is given by,

$$
J_{s}=Z \beta^{*} N_{o} e^{-W_{R}^{*} / k_{B} T}
$$

where $Z$ is the Zeldovitch factor, $\beta^{*}$ is the rate of atom impingement, $N_{o}$ is the number of atoms per volume, $W_{R}^{*}$ is the work on forming the critical nucleus, $k_{B}$ is Boltzmann's constant, and $T$ is temperature. The work on forming the critical nucleus is

$$
W_{R}^{*}=\frac{16 \pi \sigma^{3}}{3\left(\Delta G / V_{m}^{\beta}\right)^{2}}
$$

where $\sigma$ is the interfacial energy, $\Delta G$ is the volume free energy change on forming a nucleus of critical composition, and $V_{m}^{\beta}$ is the molar volume of the $\beta$ phase. The Zeldovitch factor is,

$$
Z=\left[\frac{\omega_{a}^{2} \sigma}{4 \pi^{2} k_{B} T R_{c}^{4}}\right]^{1 / 2}
$$


where $\omega_{a}$ is the volume per atom in $\alpha$, and $k_{B}$ is Boltzmann's constant.

The form of the expression for the impingement rate depends on the rate limiting mass transport process. One limit is where the impingement rate is controlled by the rate of atom motion across the interface. The other extreme is where the impingement rate is controlled by long range diffusion to a cluster. Here we examine the limit where long range diffusion of atoms to a cluster sets the impingement rate. Thus the impingement rate can be determined by solving a diffusion equation for the flux of solute atoms to a cluster. This will allow us to determine, for example, the effects of slow diffusing species on the kinetics of cluster growth. Once this is known, the growth rate of the cluster is specified and thus the impingement rate is given by,

$$
\beta^{*}=4 \pi R^{2} N_{a} \rho d R / d t
$$

where $R$ is the critical radius, $\rho$ is the constant density of the solid (moles/volume), $N_{a}$ is Avogadro's number, $d R / d t$ is the growth rate of a cluster of the critical size. In this case we neglect the corrections to $d R / d t$ due to capillarity and obtain using Eq. (4),

$$
\beta^{*}=4 \pi R N_{a} \rho \frac{\overline{\Delta C_{i}} G_{i j}^{\alpha} \Delta C_{j}^{\infty}+\bar{C}_{\delta}^{\beta}\left(\bar{\mu}_{\delta}^{\alpha}-\bar{\mu}_{\delta}^{\beta}\right)}{\overline{\Delta C}_{i} G_{i j}^{\alpha} D_{j k}^{-1} \mathcal{B}_{k}}
$$

where $\mathcal{B}_{k}$ is given in Eq. (5) with

$$
L_{k}=G_{j k}^{\beta-1}\left(\tilde{\mu}_{j}^{\alpha}-\tilde{\mu}_{j}^{\beta}\right)
$$

In the limit that the growth of the phase is limited by long range diffusion (interface kinetics limited nucleation will be included in future extension), The incubation time is

$$
\tau=\frac{1}{\theta \beta^{*} Z^{2}}
$$

where $2 \leq \theta \leq 4 \pi$.

Non-isothermal nucleation An exact calculation of the incubation time for nucleation requires a solution to the Fokker-Planck equation. In the spirit of our past work, we shall develop an approximate theory. For isothermal nucleation the incubation time $\tau$ is given by Eq. (20). The incubation time can be computed by considering the characteristic time required for a nucleus to random walk in cluster size space a distance given by the number of atoms in a cluster $1 k T$ from the top of the $W_{R}$ curve.

Since a random walk is described by a diffusion equation, it is not surprising that the Fokker-Planck equation in the region where $\partial W_{R} / \partial n$ is small (near the top of the $W_{R}$ curve where the derivative of $W_{R}$ is small) is in fact a diffusion equation. Using a solution to a diffusion equation where we allow for temperature dependent $\beta^{*}$ and $Z$, we find that the incubation time during a continuous cool, where $\beta$ and $Z$ will vary with temperature and thus time is given by,

$$
\int_{0}^{\tau} \beta^{*}\left(t^{\prime}\right) d t^{\prime}=\frac{1}{\pi Z^{2}(\tau)}
$$

This is an integral equation for the incubation time $\tau$. The Zeldovitch factor varies with temperature and hence time. By design, Eq. (16) will not yield the steady state nucleation current as $t \rightarrow \infty$, since we have neglected terms related to the gradient of $W_{R}$, but only an estimate of $\tau$.

\section{Mass Conservation}

The atoms of component $i$ are divided between the $\beta$ and $\alpha$ phases. Thus,

$$
C_{i}^{o}=(1-\phi) C_{i}^{\alpha}+\phi \tilde{C}_{i}^{\beta}
$$

where $C_{i}^{o}$ is the mole fraction of $i$ in the alloy, $\phi=n_{i}^{\beta} / n_{i}^{o}$, $n_{i}^{o}$ is the number of atoms of $i$ in the alloy, $n_{i}^{\beta}$ is the number of atoms of $i$ in the $\beta$ phase, $C_{i}^{\alpha}$ is the mole fraction of $i$ in the $\alpha$ phase, and $\tilde{C}_{i}^{\beta}$ is the mole fraction of $i$ in the $\beta$ phase. We shall approximate $\phi$ as the volume fraction of $\beta$, which is exact if the densities of $\alpha$ and $\beta$ are equal. Within the quasistationary approximation $C_{i}^{\alpha}=C_{i}^{\infty}$. Thus the mass conservation equation is,

$$
C_{i}^{o}=(1-\phi) C_{i}^{\infty}+\phi \tilde{C}_{i}^{\beta}
$$

The composition in the precipitate at the matrixprecipitate interface is a function of the radius of the particle due to capillarity and, since the alloy contains more than two components, the diffusion coefficient tensor and the supersaturation,

$$
\begin{aligned}
\tilde{C}_{i}^{\beta}= & \bar{C}_{i}^{\beta}+G_{i k}^{\beta-1} G_{k l}^{\alpha}\left[C_{l}^{\infty}-\bar{C}_{l}^{\alpha}-\frac{D_{i k}^{-1} \overline{\Delta C_{k}}}{\overline{\Delta C_{i} G_{i j}^{\alpha} D_{j k}^{-1} \mathcal{B}_{k}}}\right. \\
& \left.\left(\overline{\Delta C}_{i} G_{i j}^{\alpha} \Delta C_{j}^{\infty}+\bar{C}_{\delta}^{\beta}\left(\bar{\mu}_{\delta}^{\alpha}-\bar{\mu}_{\delta}^{\beta}\right)-\frac{8 \pi \sigma V_{m}^{\beta} f_{2}}{3 \phi}\right)\right] \\
& -\frac{8 \pi \sigma f_{2}}{3 \phi} G_{i k}^{\beta-1} \overline{\Delta V_{k}}
\end{aligned}
$$

where $f_{2}$ is the second moment of the particle size distribution and $\mathcal{B}_{k}$ is given in Eq. (5) with $L_{k}$ given in Eq. (19) and $y$ given in Eq. (8).

\section{Software Implementation}

\section{Numerical Schemes}

There is no general analytical solution available to the mathematical equations of PrecipiCalc's multicomponent precipitation models described in previous sections. Utilization of numerical methods within computer software is 
hence necessary. PSD of particle size and number density distribution is discretized in both particle size and time. The continuity equation (Eq. (3)) describing the evolution of PSD is solved in PrecipiCalc with the characteristic methods [16], and an adaptive discretization framework to provide accurate and efficient computation. To provide efficiency, the thermodynamics/diffusivity quantities are not calculated at every timestep, and are done only when the departure of current temperature and matrix composition exceeds certain limits from the state where expansion is performed.

\section{CALPHAD Tool Integration}

A unique feature of PrecipiCalc software is the tight integration of CALPHAD thermodynamics and mobility data and tools. In PrecipiCalc software, TCAPI (ThermoCalc ${ }^{\circledR}$ Advanced Programming Interface) [17] library is selected to efficiently provide all the necessary thermodynamics and diffusivity calculations. Though there are other CALPHAD software tools available, they do not currently meet the PrecipiCalc model requirements, in particular the needs of driving forces, second derivative matrices of free energies, and diffusivity matrices for multicomponent systems. Before the availability of TCAPI library, an earlier version of PrecipiCalc relied on a systemlevel layer based on UNIX IPC (Inter-Process Communication) to communicate with an external DICTRA process. However, this approach involves the system level communication overhead, and produces non-standalone user programs. In literature, Serriere et al. [8] have utilized Thermo-Calc for the precipitation simulation of binary system, while using simple representation for diffusivity without using DICTRA and atomic mobility.

\section{Software Engineering and AIM Integration}

PrecipiCalc software was mostly written in $\mathrm{C}++$ programming language to take advantage of many modern objectoriented features. In addition, several $\mathrm{C}$ and FORTRAN libraries are used, such as BLAS [18] and LAPACK [19] for linear algebra calculation, and Hierarchical Data Format (HDF) library [20] for data storage. PrecipiCalc is available for both Linux and MS Windows ${ }^{\circledR}$ platforms. The user interface of PrecipiCalc calculation program and its post-processor are text-based, and currently without a graphical user interface (GUI). The post-processor can be used to quickly produce plots for fast visualization of calculation results. The text-based PrecipiCalc interface, though not as friendly for interactive operation as a GUI, does provide a simple mechanism for higher level integration discussed later.

One of the critical components in the AIM initiative was the ability to perform the mechanistic computations of spatial variation and uncertainty/error propaga- tion analysis, which relies heavily on extensive processmicrostructure-property calculation. For the applications to aeroturbine disk alloy, the PrecipiCalc software acted as a process-microstructure simulation tool. Combined with SFTC's FEM-based DEFORM [21]-HT (Heat Treat module) as the heat treatment process simulation tool to determine the temperature evolution history at every location in a part, and the strength model developed by University of Michigan [22] for calculation of yield strength at room and elevated temperatures, these tools complete the process-microstructure-property calculation cycles. Furthermore, a CALPHAD-based APB (Anti-Phase Boundary) energy model [23] (needed by the strength model) was used to calculate APB energies for three different distributions of $\gamma^{\prime}$ particles based on the compositions output from PrecipiCalc software. In addition, a grain pinning model [24] is also used to calculate the grain size of $\gamma$ matrix based on the $\gamma^{\prime}$ particle size after the solution treatment.

Under the AIM program, a high level multisite distributed integration was achieved using the iSIGHT [25] engineering design/optimization/automation tool and secure shell utilities for networking communication. The text-based interface of PrecipiCalc allows easy integration into a script and iSIGHT to facilitate the massive automation. The overall integration frame work is shown in Figure 2.

\section{Applications to IN100 Aeroturbine Disk Alloy}

Applications of PrecipiCalc to the $\gamma^{\prime}$ microstructure simulation for heat treatment of Ni-based IN100 utilize several sources of data, which are described below.

- Multicomponent thermodynamics database - is based on ThermoTech's Ni-Data version 4.0 [26] with additional assessment of vanadium [27] under the AIM program. The IN100 compositions for $\gamma / \gamma^{\prime}$ simulation were obtained by excluding higher temperature carbides and borides from overall compositions, based on the equilibrium calculation at solution temperature. The resulting overall $\gamma / \gamma^{\prime}$ composition is $\mathrm{Ni}-13.02 \mathrm{Cr}-17.03 \mathrm{Co}-4.55 \mathrm{Ti}-10.0 \mathrm{Al}-1.86 \mathrm{Mo}-0.778 \mathrm{~V}$ in at\%. Based on this composition, the three-step sequential equilibria calculation [27] yields 19.1\%, $27.6 \%$, and $13.6 \%$ for primary, secondary, and tertiary $\gamma^{\prime}$, respectively, with a total of about $60 \%$.

- Multicomponent mobility database - is based on NIST Ni-based mobility database [28] for $\gamma$ matrix. All the relevant components of IN100 alloy are available in this database, and the full $6 \times 6$ (without $\mathrm{Ni}$ ) diffusivity matrices for $\gamma$ matrix at different temperatures and matrix compositions were used in the PrecipiCalc simulation. 


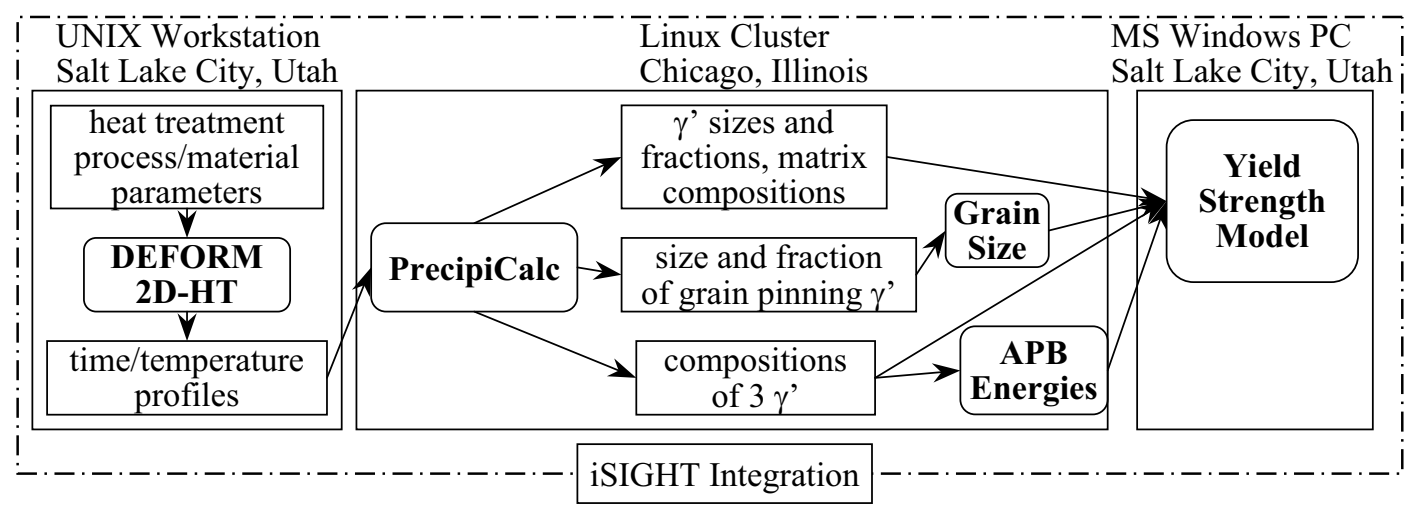

Figure 2: Distributed iSIGHT integration for mechanistic process-microstructure-property simulations, performed on different computer hardware and operating systems across network.

- Molar volume of $\gamma^{\prime}$ - is based on a multicomponent and temperature dependent model [29] established under the support of the AIM program.

- Interfacial mobility - of low coherency interface is described based on massive transformation kinetics with an activation energy scaled to that for solvent self diffusion. This was applied only to primary $\gamma^{\prime}$.

- $\gamma^{\prime}$ microstructure before the heat treatment cycle - is a necessary input to start the PrecipiCalc heat treatment simulation. Experimental information from forging was employed which gave a starting $\gamma^{\prime}$ distribution with mean diameter of $0.4 \mu \mathrm{m}$ and fraction of $43 \%$.

Instead of applying PrecipiCalc to the entire 3-step heat treatment cycle with one single calculation, a sequential three-step simulation is used for IN100 applications. The first (and second) step ends when the secondary (and tertiary) $\gamma^{\prime}$ starts to come out during the quench right after the first (and second) treatment, and calculation restarts with primary (and secondary) $\gamma^{\prime}$ particles removed. This is necessary for two reasons. First, the IN100 PrecipiCalc calculations used uniform particle compositions in the mass balance calculation and the precipitate compositions automatically follow the thermodynamics description. However, during the fast quench after the first and second heat treatments and with relatively slow diffusion in $\gamma^{\prime}$ particles, primary and secondary $\gamma^{\prime}$ particles do not have enough time to adjust and relax their compositions. Removing primary and secondary $\gamma^{\prime}$ particles during the first and second quenches prevent incorrect particle (and hence matrix) compositions for the subsequent calculations. Secondly, mean field approximation utilized by PrecipiCalc particle growth model assumes every particle interacts with all others through a single composition pool. This is a good assumption for unimodal particle size distribution where no significant size difference exists.
For multiple distributions where a large particle is surrounded by many small particles, the large particle only interacts with a layer of smaller ones around it. Applying mean field approximation hence overestimates the growth of large particles and the dissolution of small particles, yielding faster coarsening kinetics to this multimodel distribution than reality. As $\gamma^{\prime}$ particles in IN100 have three distinct size scales, with many secondary (tertiary) particles around a single primary (secondary) particle, utilizing the multistep simulation prevents the over-coarsening problem.

\section{Simulations for the Disk Center Location}

The microstructure of a center location of a full scale disk has been fully characterized at University of Connecticut under the AIM program and is used to calibrate four selected PrecipiCalc tuning parameters. An optimization to the above PrecipiCalc parameters based on available experimental information of $\gamma^{\prime}$ sizes and fractions was performed with the help of iSIGHT. Lower optimization weighting was placed for tertiary microstructure than those for primary and secondary microstructure. These tuning parameters and their determined values are listed below.

- Surface energy of primary $\gamma^{\prime}\left(0.12 \mathrm{~J} / \mathrm{m}^{2}\right)$ - represents the incoherent (due to large primary size) $\gamma / \gamma^{\prime}$ interface.

- Surface energy of secondary/tertiary $\gamma^{\prime}\left(0.061 \mathrm{~J} / \mathrm{m}^{2}\right)$ - represents the coherent $\gamma / \gamma^{\prime}$ interface. The determined value is on the high side compared to other literature studies based on coarsening kinetics of $\gamma^{\prime}$ particles.

- Diffusivity scaling factor (0.964) — is a positive value multiplied to all diffusivity matrix components to account for possible inaccuracy in the mobility 

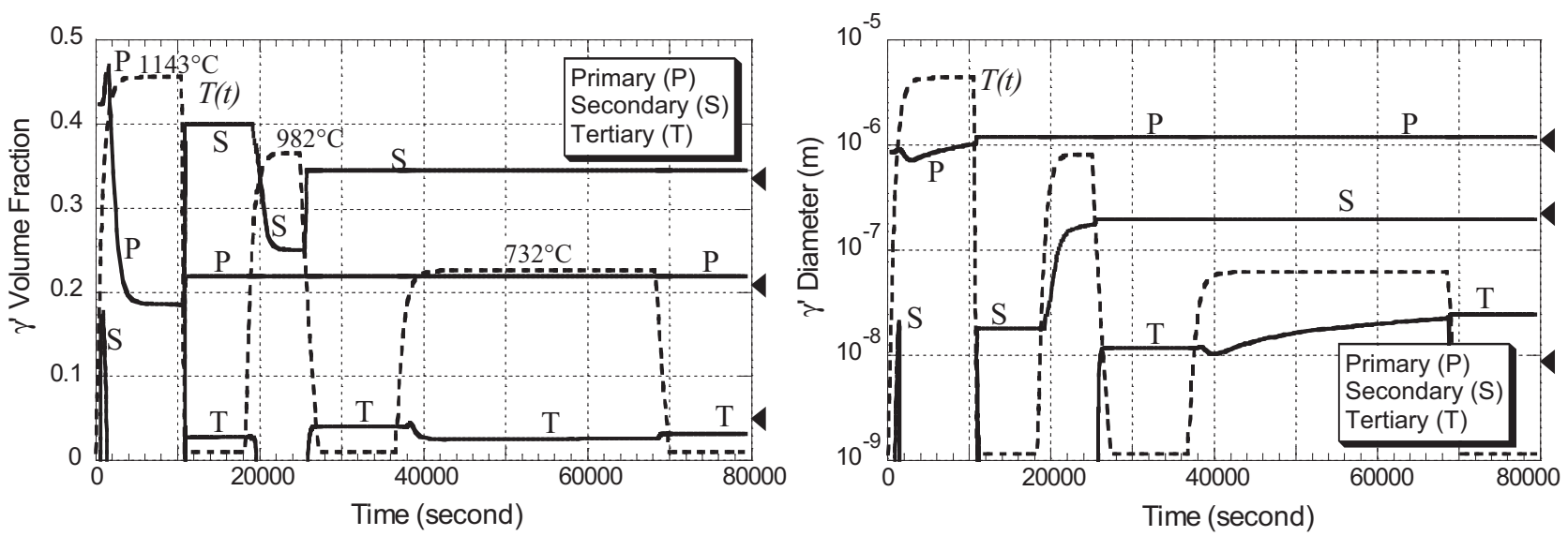

Figure 3: PrecipiCalc simulation of a center location of an aeroturbine disk under the 3-step heat treatment cycle. Time evolutions of mean sizes and fractions of three $\gamma^{\prime}$ distributions are shown with overlapped temperature profile. Experimental $\gamma^{\prime}$ microstructural results after heat treatment are shown as triangles on the right of both plots.

database. A number close to unity was found, indicating the NIST mobility database provides accurate predictions and needs little correction.

- Interfacial mobility prefactor for primary $\gamma^{\prime}(0.546$ $\mathrm{m} / \mathrm{sec}$ ) - determines the level of interfacial dissipation due to the low-coherency interface between primary $\gamma^{\prime}$ and matrix. For coherent secondary and tertiary $\gamma^{\prime}$, interfacial dissipation is considered insignificant.

Using the above optimized tuning parameters, the results of $\gamma^{\prime}$ simulation for the disk center are shown in Figure 3, where time evolutions of both mean $\gamma^{\prime}$ sizes and fractions of individual distributions are shown along with the heat treatment temperature profile overlapped. Labels "P", "S", and "T" are placed to denote primary, secondary and tertiary $\gamma^{\prime}$ kinetics, respectively. The following summarizes the major observations of this PrecipiCalc simulation.

- Multimodal $\gamma^{\prime}$ distributions are resulted from the cyclic heat treatment. The high temperature aging puts back some $\gamma^{\prime}$ formers to solution and coarsens up existing particles. The subsequent quench releases remaining supersaturation into a new nucleation, which at lower temperature with lower diffusivity, requires higher driving force to activate, leading to a small size scale in the new precipitation.

- The final $\gamma^{\prime}$ microstructure after the heat treatment observed experimentally are shown as triangles in Figure 3. PrecipiCalc simulation results with optimized parameters has provided very good agreement with experimental results except tertiary microstructure, where the calculated mean size $(20 \mathrm{~nm})$ is twice larger than that determined experimentally $(8.6 \mathrm{~nm})$.
Note that experimental observation has shown a big spread of tertiary size with maximum size found to be $17 \mathrm{~nm}$. The experimentally estimated tertiary fraction $(5 \%$ which is back determined from overall fraction and the observed primary/secondary fractions), has high uncertainty and is slightly higher than the calculated value $(3 \%)$.

- The equilibrium fractions of $\gamma^{\prime}$ are reached at three holding temperatures, comparing to the sequential equilibria calculation. For example, at second heat treatment, a total (primary+secondary) of about $57 \%$ of $\gamma^{\prime}$ is found for both PrecipiCalc and sequential equilibria results. However, after isothermal treatments, the temperature drop pushes coarsening $\gamma^{\prime}$ particles into growing particles during the quench. This introduced additional fraction increases, and led to higher primary and secondary fractions than those calculated from sequential equilibria. The final tertiary fraction from PrecipiCalc simulation ended up smaller than that from sequential equilibria results, as the total $\gamma^{\prime}$ fraction is still around $60 \%$.

- With further undercooling and more accumulated $\gamma^{\prime}$ supersaturation during the quench, new nucleation occurs and finer secondary (tertiary) $\gamma^{\prime}$ particles started to come out. The amount of finer $\gamma^{\prime}$ can achieve depends on how much $\gamma^{\prime}$ has formed previously.

- The simulated results have shown several transient and temporal $\gamma^{\prime}$ precipitations that may come out at low temperature during heat up, due to remaining and unused supersaturation of $\gamma^{\prime}$. Those transient $\gamma^{\prime}$ particles become subcritical and disappear when temperature goes up further. 


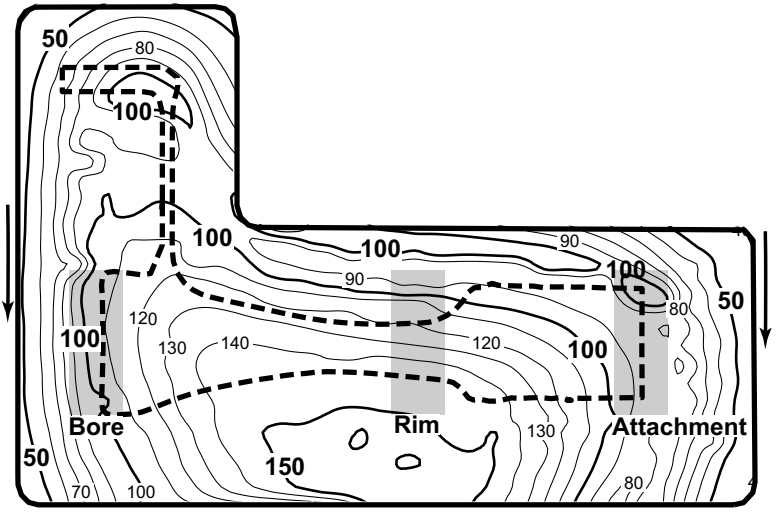

Figure 4: Calculated PrecipiCalc spatial contours of secondary $\gamma^{\prime}$ size (in $\mathrm{nm}$ ) for a radially symmetric crosssection of the minidisk. The left and right sides of the cross sections represent the inside and outside diameters, respectively. During the oil quench, the disk is immersed into quench media indicated by the arrows.

The calculated compositions of $\gamma$ and $\gamma^{\prime}$ are also compared with the experimental measurements with EDS and 3DAP (3D Atomic Probe) [30]. The 3DAP removed a total of 3 million atoms, in a box size of $12 \times 12 \times 476 \mathrm{~nm}^{3}$ along the direction of $<110>$. The box cuts through two secondary and one $\gamma^{\prime}$ particles, and two matrix channels between secondary and tertiary particles. The results are summarized in Table 1. The calculated $\gamma^{\prime}$ compositions captured the correct trends for most alloying components from high temperature $\gamma^{\prime}$ (primary) to low temperature $\gamma^{\prime}$ (tertiary), when compared to the experiments. However, deviations in some elements are evident. For the compositions of $\gamma^{\prime}$ particles, PrecipiCalc under-estimated the amounts of Mo, $\mathrm{V}$ and $\mathrm{Cr}$, and over-estimated the amounts of $\mathrm{Al}$ and Co. This is caused by the inaccuracy of the thermodynamics information used in simulation.

\section{Spatial Calculations for IN100 Disk}

Based on the experience of IN100 simulation of one location, we applied PrecipiCalc to compute the spatial $\gamma^{\prime}$ microstructure distribution of an entire heat-treated minidisk (a scaled down aeroturbine disk for testing purposes), including the integral which will be machined off after heat treatment. Using the DEFORM calculated time-temperature profiles of IN100 minidisk, we applied PrecipiCalc calculations to every FEM node. The results of PrecipiCalc were used to calculate $\gamma$ grain sizes, APB energies and yield strength, based on the software integration shown in Figure 2. All the input and parameters to PrecipiCalc remain the same as the previous disk center calculation, except for different thermal profiles depending on location, and the initial $\gamma^{\prime}$ size distribution to start the heat treat simulation has $40 \%$ larger mean

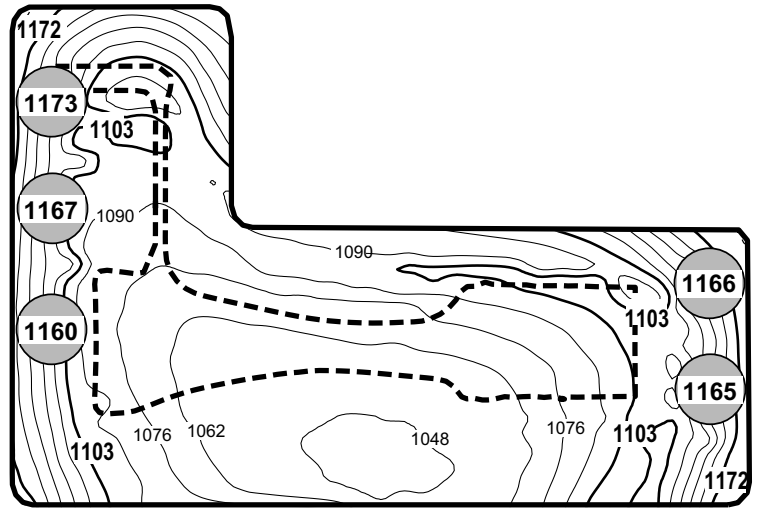

Figure 5: Calculated spatial contour of yield strength (in $\mathrm{MPa}$ ) at $260^{\circ} \mathrm{C}$ for minidisk.

size than that used in previous single location calculation. The adjustment to the initial size distribution is necessary to yield reasonable final primary $\gamma^{\prime}$ sizes observed experimentally. This adjustment falls within the microstructural variations caused by the variations of extrusion/forging conditions.

In the plot of a cross section of the minidisk with integral shown in Figure 4, the calculated secondary $\gamma^{\prime}$ size, which is the most sensitive $\gamma^{\prime}$ microstructure feature with respect to the thermal profile (particularly quench rate), is presented as a contour plot. The thick dashed line overlaid on the contour plot represents the minidisk shape machined out after the heat treatment. As indicated from the contours, the secondary $\gamma^{\prime}$ size varies from $40 \mathrm{~nm}$ at the vertical surface, to $160 \mathrm{~nm}$ at the bottom center portion of the disk. This is expected, as there is fastest cooling (smallest $\gamma^{\prime}$ ) at the vertical surface, and slowest cooling (largest $\gamma^{\prime}$ ) at the bottom surface with heat trap. The simulations were then compared to the experimental measurements at three areas: bore, rim and attachment, indicated in Figure 4 with shaded rectangles. Based on PrecipiCalc results, the secondary $\gamma^{\prime}$ sizes are fairly uniform in the vertical direction within the bore and attachment. In the rim area, however, the secondary $\gamma^{\prime}$ sizes can vary dramatically along the $y$-direction. To provide the comparison of simulation and experiments, microstructure results are summarized in Table 2. Multiple experimental values are given when different minidisks were used in characterization. A large variation in the experimentally measured secondary size was observed between different disks. Multiple PrecipiCalc values at rim location were from three points with the same $\mathrm{x}$-coordinate, but different y-coordinate, to represent the range of variation at that location. Overall, the PrecipiCalc calculations captured the correct trend in secondary size at different locations. The volume fractions of primary and secondary $\gamma^{\prime}$ are within $2.5 \%$ of measurements, within the experimen- 


\begin{tabular}{|c|c||c|c|c|c|c|c|c|}
\hline \multicolumn{2}{|c||}{ at\% } & $\mathrm{Ni}$ & $\mathrm{Co}$ & $\mathrm{Mo}$ & $\mathrm{V}$ & $\mathrm{Cr}$ & $\mathrm{Al}$ & $\mathrm{Ti}$ \\
\hline \hline \multirow{2}{*}{ Primary } & EDS & 63.1 & 11.06 & 0.84 & 0.74 & 3.73 & 12.4 & 8 \\
\cline { 2 - 9 } & PpC & 61.1 & 12.1 & 0.38 & 0.27 & 3.14 & 14.14 & 8.83 \\
\hline \hline \multirow{3}{*}{ Secondary } & 3DAP & 62.83 & 10.06 & 0.945 & 0.725 & 2.82 & 14.65 & 7.88 \\
\cline { 2 - 9 } & PpC & 63.22 & 10.66 & 0.507 & 0.568 & 2.72 & 15.25 & 7.06 \\
\hline \hline \multirow{2}{*}{ Tertiary } & 3DAP & 63.12 & 9.1 & 1.885 & 1.712 & 2.49 & 16.31 & 5.27 \\
\cline { 2 - 8 } & PpC & 64.61 & 9.32 & 1.104 & 1.14 & 2.99 & 17.18 & 3.65 \\
\hline \hline Matrix & 3DAP & 32.03 & 30.78 & 3.415 & 0.86 & 30.53 & 2.09 & 0.21 \\
\cline { 2 - 8 } & PpC & 37.51 & 26.14 & 3.841 & 1.3 & 28.38 & 2.71 & 0.11 \\
\hline
\end{tabular}

Table 1: Comparison of compositions from PrecipiCalc $(\mathrm{PpC})$ and experimental results for a disk central location.

tal accuracy. All other calculated microstructural features compare reasonably well with experiments, including tertiary sizes.

Using the strength model [22] developed under the AIM program, we also calculated the $260^{\circ} \mathrm{C}$ yield strength distribution as shown in the contour plot of Figure 5. The experimental tensile data at five selected locations are shown as five circles on the plot, representing the grip locations of the tensile bars. The experimental yield strengths are around $1166 \mathrm{MPa}$, in good agreement with predictions.

Additional Applications of PrecipiCalc in AIM Program

In addition to the previous applications to the IN100 heat treatment simulation, PrecipiCalc was utilized to model the variation of $\gamma^{\prime}$ microstructure in extrusion and forging, based on available information of processing variations in time and temperature. The results agreed very well with experiments. Furthermore, we also had preliminary simulation of both carbides and $\gamma^{\prime}$ precipitation at different solution treatment temperatures using the multiphase feature of PrecipiCalc. Combining with the distributed grain size model, we computed the grain sizes and clearly demonstrated the grain pinning control from $\gamma^{\prime}$ particles to carbides with increasing solution temperatures [24] when passing through the $\gamma^{\prime}$ solvus temperature. The high-fidelity PrecipiCalc $\gamma^{\prime}$ precipitation simulations and structure/property provided a mechanistic base for probabilistic methodology of accelerated prediction of minimum property, which was demonstrated in the AIM program.

\section{Conclusions}

Under the AIM initiative, PrecipiCalc has been greatly improved and enhanced. With the mechanistic multicomponent precipitation models of integrated treatment of nucleation, growth and coarsening, non-isothermal treatment and multiphase capability, PrecipiCalc has shown to be a useful tool for the simulations of commercial alloy processing. The successful application of PrecipiCalc to the $\gamma / \gamma^{\prime}$ simulation of the IN100 Ni-based aeroturbine disk alloy under commercial heat-treatment processing is discussed in this article. The particular emphasis here is the spatial microstructure and yield strength prediction in the entire disk, with the integration of processing and property models and tools. With this mechanisitc microstructure prediction tool, it is possible to address the property variation with other statistical models and tools developed under the AIM initiative.

Though PrecipiCalc was originally developed to capture the $\gamma / \gamma^{\prime}$ precipitation in Ni-based superalloys, it was built with mechanistic CALPHAD-based models, allowing quick applications to other alloy systems. For example, in other government funded projects, PrecipiCalc has been used to simulate the strengthening dispersion such as Heusler phase in NiTi alloys and carbides/intermetallics in steels, the grain pinning dispersion in heat affected zone of welding, and the devitrification kinetics from amorphous iron/aluminum alloys.

Many modeling enhancements for PrecipiCalc are currently under development, including heterogeneous nucleation (on different types of defects and catalyst particles), denuded zone to provide an estimate of the size of solute depleted zones associated with growth, precipitation under constrained equilibrium.

\section{Acknowledgments}

This work was supported by DARPA/USAF under Contract No. F33615-00-2-5216 and under subcontract from Pratt \& Whitney. The authors acknowledge Pratt \& Whitney, Martin Blackburn of University of Connecticut and Gautam Ghosh of Northwestern University for providing the experimental results, Tresa Pollock of University of Michigan for the strength model, and Mike Long of Engineous Software for performing the iSIGHT integrated calculations. The authors would also like to thank Ryan Rathbun, Lei Li and Christina Scharer of QuesTek for their support in software implementations and applications, and additional support provided by State of Illinois Technology Challenge Grant, No. 02-49106. 


\begin{tabular}{|c|c|c|c|c|c|c|c|}
\hline \multirow{2}{*}{\multicolumn{2}{|c|}{ Minidisk Comparison }} & \multicolumn{2}{|c|}{ Bore } & \multicolumn{2}{|c|}{ Rim } & \multicolumn{2}{|c|}{ Attachment } \\
\hline & & Exp. & $\mathrm{PpC}$ & Exp. & $\mathrm{PpC}$ & Exp. & $\mathrm{PpC}$ \\
\hline \multirow[t]{2}{*}{ Primary $\gamma^{\prime}$} & Fraction (\%) & $\begin{array}{c}24 \\
25.2\end{array}$ & 22.6 & $\begin{array}{c}23.5 \\
25\end{array}$ & 23.5 & $\begin{array}{l}23.1 \\
25.7\end{array}$ & 23.3 \\
\hline & $\operatorname{Size}(\mu \mathrm{m})$ & 1.28 & 1.29 & $\begin{array}{l}1.23 \\
1.27\end{array}$ & 1.32 & $\begin{array}{c}1.18 \\
1.2\end{array}$ & 1.31 \\
\hline \multirow[t]{2}{*}{$\overline{\text { Secondary } \gamma^{\prime}}$} & Fraction (\%) & 32.4 & 35 & & 34 & & 34.6 \\
\hline & Size(nm) & $\begin{array}{l}109 \\
129\end{array}$ & 107.9 & $\begin{array}{l}132 \\
157\end{array}$ & $\begin{array}{l}120 \\
135 \\
146\end{array}$ & $\begin{array}{l}103 \\
114\end{array}$ & 84.2 \\
\hline Tertiary $\gamma^{\prime}$ & Size $(\mathrm{nm})$ & $\begin{array}{c}18 \\
20.8\end{array}$ & 21.5 & $\begin{array}{l}19.7 \\
21.8\end{array}$ & 21.4 & 21.4 & 20.7 \\
\hline
\end{tabular}

Table 2: Minidisk microstructure prediction from PrecipiCalc and experimental results

\section{References}

[1] J.S. Langer and A.J. Schwartz, Physical Review A, $21(1980)$ 948-958.

[2] R. Kampmann and R. Wagner, Materials Science and Technology, vol.5. Germany: VCH Weinheim, (1991) 213-303.

[3] I.M. Lifshitz and V.V. Slyozov, Phys. Chem. Solids, 19 (1961) 35.

[4] C. Wagner, Z. Electrochem., 65 (1961) 581.

[5] A. Deschamps and Y. Brechet, Acta mater., 47 (1999) 293-305.

[6] J.D. Robson, M.J. Jones, and P.B. Prangnell, Acta Materialia 51 (2003) 1453-1468.

[7] R. Kampmann and R. Wagner, presented at Decomposition of Alloys: the Early Stages, Sonnenberg, Germany, 1983.

[8] M. Serriere et al., Materials Science Forum, 396-402 (2002) 747-752.

[9] V.V. Popov, Phys. Met. Metallogr., 93 (2003) 303309.

[10] V.V. Popov and I.I. Gorbachev, The Physics of Metals and Metallography, 95 (2003), 417-426.

[11] P.E.J. Rivera-Díaz-del-Castillo, Scripta Mater., 47 (2003) 113-117.

[12] C.J. Kuehmann and P.W. Voorhees, Metal. Trans. A., 27 (1996) 937-943.

[13] J. Morrel and G. Purdy, Scripta Mater., 30 (1994) 905-908.

[14] J. Agron et al., CALPHAD, 204 (2000) 42-54.
[15] A. Umantsev and G.B. Olson, Scripta Mater., 29 (1993) 1135-1140.

[16] S. Kumar and D. Ramkrishna, Chemical Engineering Science, 52 (1997) 4659-4679.

[17] http://www.thermocalc.com/

[18] J.J. Dongarra et al., ACM Trans. Math. Soft., 16 (1990) 18-28.

[19] E. Anderson et al., LAPACK Users' Guide, 3rd ed., Society for Industrial and Applied Mathematics, (1999), Philadelphia, PA.

[20] http://hdf.ncsa.uiuc.edu/HDF5/

[21] http://www.deform.com/

[22] R. Kozar, J. Schirra, W. Milligan and T.M. Pollock, submitted to Metall. Trans.

[23] A.P. Miodownik and N. Saunders, Applications of Thermodynamics in the Synthesis and Processing of Materials, ed. Nash and Sundman, TMS, (1995) 91.

[24] J. Wright and R. Rathbun, to be published.

[25] http://www.engineous.com/

[26] N. Saunders, M. Fahrmann and C.J. Small, in Superalloys 2000, eds. K.A. Green, T.M. Pollock and R.D. Kissinger, (TMS, Warrendale, 2000), 803.

[27] A.M. Wusatowska-Sarnet et al., J. Mater. Res., 18 (2003) 2653-2663.

[28] C.E. Campbell, W.J. Boettinger, and U.R. Kattner, Acta Materialia 50 (2002) 775-792.

[29] H.-J. Jou, unpublished work performed under DARPA-AIM program, 2000.

[30] D. Isheim, to be published. 